\title{
THE SCHÖNLEIN-HENOCH SYNDROME WITH PARTICULAR REFERENCE TO RENAL SEQUELAE
}

\author{
BY \\ R. J. DERHAM and M. M. ROGERSON \\ From the Department of Child Health, Alder Hey. Children's Hospital, Liverpool
}

(RECEIVED FOR PUBLICATION MAY 11, 1956)

In recent years the Schönlein-Henoch syndrome has been discussed in many articles and particular interest has centred around the incidence and outcome of renal complications. As long ago as $\mathbf{1 8 9 9}$ Henoch stated that nephritis was a frequent complication which might progress to anasarca and prove fatal. In a recent series, Wedgwood and Klaus (1955) found that, of 26 cases followed for periods between two and 15 years, 10 children had abnormal urine, only six of these having had proteinuria or haematuria during the acute stage of the disease. These authors, therefore, believe nephritis may occur as a late sequel in some cases even when urine examinations showed no abnormality during the acute stage. On this basis it has been postulated that the Schönlein-Henoch syndrome might be considered as a possible latent cause of nephritis in older children and adults. Oliver and Barnett (1955), on the other hand, say that, although renal involvement occurs in approximately half the children with anaphylactoid purpura, their follow-up results indicate that nephritis does not emerge as a late sequel.

In 1952 we published a series of 35 typical examples of the Schönlein-Henoch syndrome, seen between the years 1943 and 1950, together with four cases which were atypical but which appeared to be related disorders of the collagen system (Derham and Rogerson, 1952). It is now proposed to review the progress of as many of the original cases as it has been possible to trace and to report a further 59 cases which have been admitted to Alder Hey Children's Hospital, Liverpool, from 1951 to 1955. The earlier cases have been reviewed in an attempt to determine the incidence of renal complications. Addis counts and urea clearance tests have been carried out in 34 patients taken from the two series, irrespective of whether they had, or did not have, renal complications at the time of their original illness.

\section{Follon -up of 1943-50 Series}

Of the original 35 typical cases admitted between 1943 and 1950, nine had haematuria and another 10 had albumin and red cells in their urine while they were still in hospital, a total of $19(54 \%)$ with renal involvement of varying degree. At the time of their discharge from hospital 15 of these children had clear urine whilst four still had albumin and red cells. Of these four children, three had no other clinical evidence of nephritis and they are now quite well. One child has had considerable albuminuria for eight years but is now recovering and recent renal function tests give normal results.

Some of the patients in this series have left the district, but 17 of the 35 have been traced. It has been found that nine of them have had no recurrence of any symptoms, five report occasional joint pains and swelling, one has had the rash on one occasion with joint swellings, pain and colic, and two have had frequent recurrences of the rash, together with abdominal pain and swelling of ankles.

Urea clearance and or Addis counts have recently been carried out for 14 of the patients, including the two children with frequent recurrences. These tests gave normal results in 10 instances. Two patients, girls of 13 and 19 years, showed a mild impairment of kidney function, one girl of 18 years had moderately impaired function and one other girl of 15 years has a severe deficit. This last case was complicated by a recent nephrectomy for hydronephrosis.

Four Atypical Cases in the 1943-50 Series. The four cases reported, which were described as 'atypical', had some of the clinical manifestations of the Schönlein-Henoch syndrome associated with other aspects of collagen disease. One child (Brian J., Case 4) had already died from periarteritis nodosa when the cases were reported. Of the remaining three cases, two are well and one has died. These three cases were as follows: 
TABLE 1

SCHÖNLEIN-HENOCH SYNDROME CASES ADMITTED TO ALDER HEY CHILDREN'S HOSPITAL, LIVERPOOL. 1943-1955

\begin{tabular}{|c|c|c|c|c|c|c|c|c|c|c|c|c|c|c|}
\hline & 1943 & 1944 & 1945 & 1946 & 1947 & 1948 & 1949 & 1950 & 1951 & 1952 & 1953 & 1954 & 1955 & Total \\
\hline $\begin{array}{l}\text { Males } \\
\text { Females }\end{array}$ & 1 & $\frac{2}{1}$ & 2 & $\begin{array}{l}1 \\
3\end{array}$ & $\begin{array}{l}4 \\
-\end{array}$ & $\frac{2}{6}$ & $\begin{array}{l}4 \\
6\end{array}$ & $\frac{2}{1}$ & $\begin{array}{l}3 \\
5\end{array}$ & $\begin{array}{l}6 \\
5\end{array}$ & $\begin{array}{r}10 \\
5\end{array}$ & $\begin{array}{l}8 \\
4\end{array}$ & 6 & $\begin{array}{l}51 \\
43\end{array}$ \\
\hline Total & 1 & 3 & 2 & 4 & 4 & 8 & 10 & 3 & 8 & 11 & 15 & 12 & 13 & 94 \\
\hline
\end{tabular}

Case 1 (Rodney McW.). This boy was $5 \frac{1}{2}$ years old when he was admitted to hospital in 1949 with haematuria, abdominal pain, vomiting, swelling of joints, a purpuric rash and a history of asthma. The rash became so necrotic that local areas of gangrene appeared. This child had a long, severe illness, lasting five months and, when discharged, still had persistent albuminuria but no hypertension. During the succeeding six years he has had five purpuric episodes and frequent asthmatic attacks. Despite the recurrences, his kidney function, as shown by urine examinations, blood urea, Addis count and urea clearance tests, is normal.

Case 2 (Jeanette D.). When first admitted to hospital in 1950, this girl had had recurring purpura on the arms and legs and joint swellings for the previous three years. She developed subcutaneous nodules while in hospital. Skin biopsies presented the histological appearance of disseminated lupus erythematosus and muscle biopsy showed panarteritis with thrombosis, recanalization and cellular infiltration of the walls of vessels. No abnormality was found in the urine. This patient is now 20 years of age and works as a cinema usherette. She is in good health, has had no recurrences, and recent kidney function tests show no abnormality.

Case 3 (Beryl R.). This girl, aged $9 \frac{1}{2}$ years, had eight episodes of abdominal colic and haematuria in 12 months. Her widespread rash resembled erythema marginatum, but the clinical picture suggested a relationship with the Schönlein-Henoch syndrome. She was in hospital for six months and, after discharge, continued to have albuminuria. Two years later she was readmitted with haematuria and developed hypertension, general oedema, ascites and pleural effusions. She died with a terminal pericarditis. At necropsy, the kidneys were large and pale. Histologically, the picture was that of a mixed Ellis type I and type II nephritis.

\section{Present Series, 1951-55}

During the five-year period, 1951-55, another 59 undoubted examples of the Schönlein-Henoch syndrome were seen in Alder Hey Children's Hospital. These, with the previous series of 35 , make a total of 94 cases. There were 51 males and 43 females (Table 1), a ratio of $1 \cdot 2: 1$, which is a slightly higher proportion of females than the ratio of 2:1 found by Oliver and Barnet (1955) and higher also than the $3: 2$ ratio in the 139 cases recorded by Lewis (1955).
In the present series the youngest child was aged 20 months and the oldest 14 years: both were girls. Nearly half the total number, however, fell into the age group 5-7 years (Table 2).

TABLE 2

SCHÖNLEIN-HENOCH SYNDROME CASES ADMITTED TO ALDER HEY CHILDRENS HOSPITAL. LIVER POOL, 1943-1955

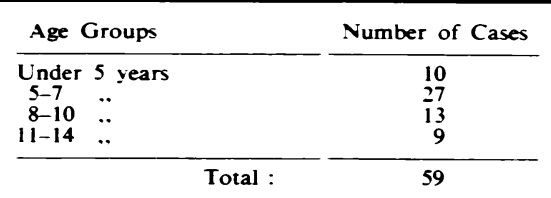

Two deaths occurred. One, a girl aged 11 years, died 10 weeks after admission from cardiac failure and chronic nephritis. She had had five recurrences of the rash and repeated attacks of colic and haematuria during her illness. Unfortunately, permission for necropsy was refused. The other child was a boy, aged 6 years, who died of uraemia 11 weeks after admission. He had had a purpuric rash, abdominal colic and joint swellings on admission, but did not have any renal complications until two weeks later, when he developed albuminuria, haematuria, anuria and hypertension, and eventually died from uraemia. Post-mortem examination was carried out in this case; the kidneys were pale and histological examination showed advanced changes of chronic glomerulonephritis.

There was no very marked seasonal incidence in the occurrence of these 59 cases of the recent series. As might be anticipated, a majority of the children were admitted during the winter months (OctoberMarch, 34 cases; April-September, 25 cases). The length of stay varied considerably, depending mainly upon the presence or absence of renal involvement. Other factors, such as recurrence of rash, joint swelling and abdominal colic and social aspects, played less important parts in prolonging their stay in hospital. The average length of stay was nine weeks, but those with gross urinary changes remained for 21 weeks (average).

Clinical Findings in the 1951-55 Series. The following are the most outstanding: 
Tonsillitis. Only 15 of the 59 patients complained that they had had a recent sore throat, but a throat swab was always taken on admission and in 20 cases haemolytic streptococci were isolated. In one child with suspected intussusception, the throat swab remained sterile but a swab taken from a laparotomy wound grew haemolytic streptococci. Group typing was carried out in only three cases, the results being: type 5 (two cases) and type 19 (one case).

RASH. The typical purpuric rash, as described by Gairdner and other writers, was present in every case, but in 19 patients it was not the first manifestation: 10 children had joint swelling and nine had abdominal pain before the rash appeared. In one case the rash was so severe that necrosis occurred.

Jornt Swellings. These occurred in 35 cases: in 20 of these more than one joint was affected, the commonest being the ankle and knee.

Abdominal Colic. Abdominal colic occurred in 30 cases. Four were submitted to laparotomy: in three the presenting symptom on admission had been abdominal pain of such severity that an acute surgical condition had been diagnosed. At operation petechial haemorrhages were seen on the intestinal walls, but in each case skin purpura appeared only after an interval of several days. The fourth case developed an intussusception one week after admission with rash and swelling of the ankles. The intussusception was reduced at operation.

In addition, two other children had signs of intestinal obstruction and abdominal radiographs showed fluid levels. Both these patients had severe colic, vomiting and haemorrhage from the bowel, but both recovered without surgical intervention.

BleEDING. Bleeding from the intestinal tract occurred in 18 cases.

Vomiting. This, as an early symptom, was present in 22 cases, and was accompanied by colic in 19 of them. Haematemesis occurred in three cases, and in one, a girl aged 7 years, vomiting was so intractable that intravenous fluids were required for three days.

Haematuria. Macroscopic haematuria occurred in 16 cases: in seven of these it was a presenting symptom and in nine it occurred after admission. In 27 cases in all, albumin, red cells, leucocytes and occasional casts were reported in the urine in one or more of the early specimens examined. Sixteen of the 27 patients had clear urine before they were discharged. Only one patient in this group was re-admitted and he relapsed with a severe, necrotic rash, but on this occasion there was no renal involvement. Of the remaining 11 cases, nine had urine which still contained albumin, red cells and casts on discharge. Two children (to whom reference has already been made) died from chronic nephritis and its complications.

Relapses and Re-admission. The tendency for patients with the Schönlein-Henoch syndrome to have a recurrence of their symptoms is well known, and 31 of our 59 patients had relapses while they were still in hospital. The causes are shown in Table 3. The commonest form of relapse was a

TABLE 3

SYMPTOMS OF RELAPSE IN 30 CASES OF THE SCHÖNLEINHENOCH SYNDROME CASES ADMITTED TO ALDER HEY CHILDREN'S HOSPITAL, LIVERPOOL, 1943-1955

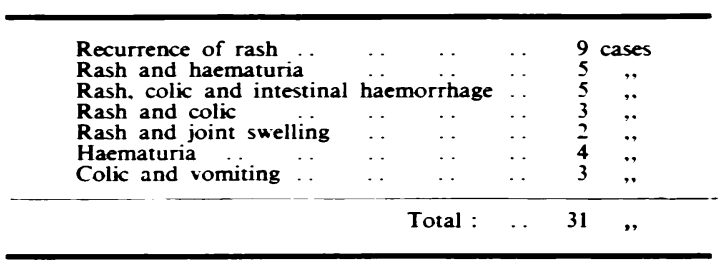

recurrence of the rash, usually as an isolated phenomenon (nine cases) but also in association with haematuria (five cases), bleeding from the bowel (five cases) and abdominal colic (three cases).

A majority of the patients attended an out-patient clinic after discharge from hospital. Five patients reported recurrences of the rash and two had had severe abdominal colic.

Ten, six males and four females, of the 59 patients in this series were re-admitted to hospital. Nine were re-admitted within four months of their discharge and one boy returned after two years. He had a recurrence of haematuria, although in the two-year interval, during which time he attended the out-patient clinic, his urine had been clear. The haematuria was profuse and recurred on several occasions during his first week in hospital, but it was his only symptom on this occasion. Previously he had had purpura, haematuria, intestinal haemorrhage and colic. He was again discharged with normal urine and renal function tests.

Of the other nine patients who were re-admitted, three had purpura and six had haematuria; four of the latter have developed chronic nephritis.

Other Complications. It is interesting that chorea was associated in two cases. One child, who had been admitted with purpura and colic, developed chorea during the convalescent period of her illness. The other child had chorea first, and one month after recovery she developed purpura and joint swellings. She now has chronic nephritis.

Asthmatic attacks were recorded in one patient, 
a boy of 13 years. He had had asthma in infancy but had been free from attacks during later childhood. An eosinophilia above $5 \%$ of the total white cell count was recorded in six cases, the figures ranging from 6 to $14 \%$.

\section{The Incidence of Renal Damage in Both Series}

In the first series of 35 cases 19 had renal involvement at the time of their initial illness and four still had red blood cells and albumin on discharge.

The general follow-up has revealed that these four patients now have normal kidney function tests, but that renal damage has occurred in four other cases. Of the four atypical cases, three developed renal complications and two of these died, one having periarteritis and the other chronic nephritis and pericarditis. The third child had persistent albuminuria for six years but now appears to have recovered.

In the second series of 59 cases, 27 patients had abnormal urinary findings at some time. Two children died from chronic nephritis. Nine others left hospital with persistent albumin and red cells in the urine; all have been followed in the Outpatient Department and five have now been discharged. The four remaining children with initial renal involvement have all developed chronic nephritis.

During the follow-up investigation it has been discovered that one other child, who did not have any evidence of renal damage at the time of the acute illness five years ago, now has impaired renal function, as shown by urea clearance test and Addis count.

In reviewing the whole series of 94 patients it has been possible to carry out renal function tests in 34 cases. Of these 22 had some renal involvement at the time of their acute illness, and 12 had no urinary abnormality. Of those who had initial renal damage, seven now show impaired kidney function. Of those who had no renal damage in the first place two patients show evidence of renal deficiency and in both cases it is of moderate severity. It is also of interest that two other children, who have had albuminuria and recurrent haematuria, for eight and seven years respectively, are now clinically well and recent renal function tests show no abnormality.

\section{Discussion}

The view has been expressed that there has been an increasing incidence of the Schönlein-Henoch syndrome over the past several years. Certainly more cases are reported now than formerly. In the five years immediately before 1948 an average of $2 \cdot 8$ cases per annum were diagnosed at Alder Hey
Children's Hospital. In 1948 the number more than doubled and, during the seven ensuing years, the average number of new cases has been 10.3 annually. Possibly this increase is ascribable in part to the interest aroused by Gairdner's valuable paper published in 1948. It is a matter for speculation whether there has been a real increase in the incidence of the syndrome, or whether awareness of the condition as a clinical entity has led to the recognition of more cases. The number of cases diagnosed at Alder Hey Children's Hospital during the past four years has remained fairly constant (Table 1).

There is general agreement that the greatest danger of the Schönlein-Henoch syndrome is renal damage. Evidence that renal damage occurred at some stage in the syndrome was found in $11(42 \%)$ of 26 cases (Oliver and Barnett, 1955) and in $19(47.5 \%)$ of 40 cases (Philpott, 1952) and in $46(49.5 \%)$ of 94 cases in the present series. Such damage was shown by albuminuria, haematuria or both, but it has not been established that these findings constitute acute glomerulonephritis. When referring to the joint manifestations of the Schönlein-Henoch syndrome, Gairdner (1948) wrote that these were not in the least like rheumatism. Is it not equally true that the initial renal involvement is not in the least like nephritis? Neither facial oedema nor hypertension are commonly found, nitrogen retention is rare, and the erythrocyte sedimentation rate tends to settle to a normal level within a short time despite continued haematuria. Wedgwood and Janeway (1953) have recorded another important difference: serum complement is normal in the Schönlein-Henoch syndrome and is markedly reduced in true nephritis.

In the present series it has been noted that leucocytes are present in the urine in greater numbers than in most cases of acute nephritis. Here, then, is an atypical picture, which might be better described as 'renal bleeding' yet, as the present series shows, this earlier involvement is a pointer to the possibility that lasting renal damage may ensue.

Clark (1956), reviewing 265 children with nephritis, has commented upon the complex and infinitely variable pictures presented, and Rubin (1955), with reference to the Schönlein-Henoch syndrome, remarked upon the 'wide pattern' of renal involvement in this disease. A study of the patients who have had renal involvement in our own series confirms this. The clinical picture of subacute nephritis (with massive albuminuria, oedema, low plasma protein, reversed albumin globulin ratio and high blood cholesterol) was seen as well as chronic nephritis with hypertension and uraemia. 
Recurrences of the syndrome, especially with renal symptoms, have been considered detrimental to the ultimate prognosis, but this assumption has not always proved correct in our series. Two patients have had several recurrences and yet their renal function tests have become normal.

In the future some help in distinguishing the types of nephritis that occur may be forthcoming from a study of electrophoresis of the serum proteins. In one of our cases the pattern of distribution of serum protein fractions corresponds to that found in the Ellis type II nephritis. It is interesting that one other case, uncomplicated by renal damage, showed an electrophoretic pattern characteristic of the early phase of acute rheumatism. The authors hope to report the result of further investigations along these lines.

\section{Summary}

From 1943 to 1955,94 examples of the SchönleinHenoch syndrome have been admitted to Alder Hey Children's Hospital, Liverpool. The incidence of renal sequelae has been investigated.

During their initial illness 46 children had some renal involvement: two children died with chronic nephritis and 13 left hospital with persistent albumin and red cells in their urine. A recent follow-up with Addis counts and urea clearance tests show that nine of these 13 children now have no evidence of kidney disease; the remaining four have developed chronic nephritis. In this series, therefore, the incidence of chronic nephritis is approximately $6 \%$.

In addition, it has been found that five other children, who did not have renal involvement at the time of their original illness and are now symptom-free, have some impairment of renal function as shown by Addis counts and urea clearance tests.

Four atypical cases described in a previous paper have been reviewed: two of these died.

Our thanks are due to Professor N. B. Capon for his helpful criticism and advice, to colleagues who kindly allowed us to include their cases in this series, and to $\mathrm{Mr}$. J. Ireland for biochemical investigations.

\section{REFERENCES}

Clark. N. S. (1956). Archives of Disease in Childhood. 31. 12. Derham. R. J. and Rogerson, M. M. (1952). Ibid.. 27, 139. Gairdner, D. (1948). Quart. J. Med., 17, 95. Henoch, E. (1899). Vorlesungen über Kinderkrankheiten, 10th ed., p. 839. Berlin.

Lewis, I. C. (1955). Archives of Disease in Childhood, 30, 212 Oliver. T. K and Barnett H. (1955) Oliver, T. K. and Barnett, H. L. (1955). Amer. J. Dis. Child.. 90. 544.
Philpott, M. G. (1952). Archives of Disease in Childhood, 27, 480. Rubin, M. I. (1955). Amer. J. Dis. Child.. 90. 545. Wedgwood, R. J. P. and Janeway, C. A. (1953). Pediatrics, 11, 569. and Klaus, M. H. (1955). Ibid., 16. 196. 